\title{
How Far could the Smoking Cessation Duration Affect Postoperative Pulmonary Complications?
}

\section{Ali $\mathrm{AM}^{1}$, Naser Al-Dossari $\mathrm{D}^{2}$, Naguib $\mathrm{NN}^{3}$, Hussein helmy $\mathrm{M}^{3}$, Ahmed Selim $\mathbf{M}^{3}$, Mickae $\mathrm{SA}^{3}$ and Nasser M Dobal ${ }^{3 *}$}

${ }^{1}$ Professor of Anesthesia at Cairo University; Chairman of Anesthesia and Intensive Care Department at Prince Sultan Military College for Health Science in Dhahran, Saudi Arabia (PSMCHS)

${ }^{2}$ Lecturer of anesthesia technology at PSMCHS in Dhahran, Saudi Arabia

${ }^{3}$ Anesthesia technology students at PSMCHS in Dhahran, Saudi Arabia

\section{Investigation Paper}

Volume 5 Issue 1

Received Date: January 16, 2020

Published Date: January 31, 2020

DOI: $10.23880 /$ accmj-16000169

*Corresponding author: Nasser M Dobal, Lecturer of anesthesia technology at PSMCHS in Dhahran, Cairo University, Saudi Arabia, Tel: 01025588779; Email: dobalnasser@yahoo.com

\section{Abstract}

Background: Smoking is a risk factor for postoperative pulmonary complications following lung resection surgery in cancer patients. The respiratory system might take 6 weeks to return to normal function after general anaesthesia for major surgery. Induction of general anaesthesia can cause changes and reductions of the respiratory parameters e.g. reduction of lung volume. A recent study by Kozub found that the duration of smoking cessation had no significant influence on the frequency of postoperative pulmonary complications, and that the optimal timing for preoperative smoking cessation has not been identified.

Purpose and aim: to evaluate the effect of the duration of smoking cessation pre-operatively and the incidence of postoperative pulmonary complications after general anaesthesia in patients with lung cancer coming for lung resection.

Design and Methodology: a retrospective observational design using patient records as a data collection tool. The population was adult smoker patients who were coming for elective lung resection surgery under general anaesthesia in King Fahad Specialist Hospital in Dammam Saudi Arabia. A sample of 30 patients was determined based on the available number of patients who met the study inclusion criteria. A chi-square test was performed to examine the association between smoker patients who stopped smoking for 6 weeks and other smoker patients who stopped smoking for less than 6 weeks prior to surgery.

Results and findings: The findings revealed that there was not statistical difference in the incidence of post-operative pulmonary complications after lung resection surgery under general anaesthesia between smoker patients who stopped smoking for more than six weeks and smoker patients who stopped smoking for less than six weeks. The Chi-square was 3.33 with a P-value of 0.07 ; showing no statistical significance between variables from the two groups. $100 \%$ of the smoker patients who stopped smoking for more than 6 weeks (15 patients of the study sample) had no post-operative pulmonary complications, and $20 \%$ of smoker patients who stopped smoking for less than 6 weeks (15 patients of the study sample) had a few post-operative pulmonary complications. However, this findings was not statically significant. Another chi-square test performed to find out the type of post-operative pulmonary complications occurred. The type of complications found were cough, bronchitis, bronchospasm and pneumonia. The onset of post-operative pulmonary complications was also evaluated 
which occurred either after 48, 50 or 72 hours in the post-operative period.

Conclusion: The No association between smoker patients who stopped smoking for 6 weeks and other smoker patients who stopped smoking for less than 6 weeks on the incidence, severity, and onset of post-operative pulmonary complications. It would seem unnecessary to postpone surgery in order to allow an optimal smoking cessation period for patient coming for lung resection surgery under GA. Smoking cessation is however still strongly recommended when treating patients with lung cancer, but it would seem unnecessary to delay surgery in order to ensure an optimal cessation of smoking period. Moreover, literature is inconsistent about the optimal time for smoking cessation before surgery. Focus on preoperative patient management, anaesthesia, postoperative pain management and surgical techniques are more effective to improve pulmonary function and reduce post-operative pulmonary complications.

Keywords: Smoking; Cancer; Lung

\section{Introduction}

The most common PO pulmonary complications are; Lung Infections: Its incidence is $20 \%$. Predisposing causes include prolonged operations and prolonged antibiotic treatment. Lung Collapse: Areas of lung tissue are prone to collapse, because of the anaesthetic and surgical problems. Pulmonary edema: Excessive fluid in the lung due to fluid overload, or heart dysfunction or as a complication of airway obstruction [1].

Aspiration pneumonia: Pulmonary inflammation caused by aspiration of stomach or other secretions (blood, vomit, food) into the lung. The lung inflammation is usually more severe if the stomach contents are acidic. Exacerbation of Asthma or Bronchitis: It is not uncommon for asthmatic or chronic bronchitis patients to suffer an attack following the operation. Pulmonary Embolism: Usually presents with shortness of breath, but sometimes there may be no symptoms. With larger emboli there is also chest pain, wheezing and lung collapse.

The respiratory system may take 6 weeks to return to its preoperative state after general anesthesia for major surgery. Risk factors for postoperative pulmonary complications development are many e.g. smoking, and anaemia [2]. Our target is to assess the relationship between the duration of smoking cessation pre-operatively and the severity of post-operative pulmonary complications. Post-operative pulmonary complications (PPCs) are pulmonary abnormality occurring between one to three days following surgery [3]. PPCs occur in 5-10\% of patients undergoing non-thoracic surgery and can reach up to $22 \%$ of high-risk patients. Even in minor surgeries, the frequency of its occurrence can be 1-2\%. Deaths related to PPCs can reach as many as one in four deaths occurring within a week of surgery. There is a wide variation in the incidence of PPCs from $2 \%$ to $40 \%$. The PPCs can vary be can be summarized as follows: Atelectasisresulting in post-operative hypoxemia (most widespread complication), pneumonia, bronchitis, bronchospasm, exacerbation of previous lung disease, pulmonary collapse due to mucus plugging of the airways, respiratory failure, acute lung injury (ALI) including aspiration pneumonitis, transfusion-related ALI (TRALI), acute respiratory distress syndrome (ARDS), and pulmonary embolism [4]. Encourage reduction or cessation of smoking at least 4 weeks and preferably 8 weeks or more before surgery. Respiratory drive and muscle function are altered, lung volumes reduced, and atelectasis develops in $>75 \%$ of patients receiving a muscle relaxant drug [5].

The research justification: There are mandatory urgent surgeries for smoker patients, in which they cannot be postponed until 6 weeks for smoking cessation [1] for example bronchogenic carcinoma. In such type of surgery, the cancer is due to smoking and surgery cannot be delayed. We were planning to compare the comparison is between smoker patients who quit smoking for 6 weeks and who quit smoking for less than 6 weeks. For example, if the percentage of chest complications in the first group as there is smoking cessation for 6 weeks is very near comparing to the cases with smoking cessation for less than 6 weeks, so it clarifies that smoking cessation for 6 weeks preoperative is not necessary. However, if there is a large difference between the percentages of pulmonary complications in both groups, then it is important to take some requirement precautions in urgent cases to decrease the postoperative pulmonary complications.

The gap of our study: We are obliged to postpone surgeries and stop smoking for less than 6 weeks. (Cases of lung carcinoma are considered as potentially emergency cases).

The importance of our study: This study shows how far the morbidity of smoking is related to the period of smoking cessation hence the precaution is put to avoid such morbidity with cases of less than 6 weeks cessation. 


\section{Literature Review}

Smoking and timing of cessation on postoperative pulmonary complications after curative-intent lung cancer surgery [5]. Smoking is a risk factor for postoperative pulmonary complications (PPCs) following non-small cell lung cancer (NSCLC) surgery. The optimal timing for preoperative smoking cessation has not been identified. Our study aimed to observe the impact of preoperative smoking cessation on PPCs incidence and other postoperative outcomes including long-term survival. A prospective study included consecutive patients following resection for NSCLC in a regional thoracic centre over a 4-year period (20102014). Patients were stratified according to self-reported preoperative smoking status. The primary endpoint was PPC incidence, which was assessed from postoperative day one onwards using the Melbourne Group Scale. Secondary endpoints included short-term outcomes (hospital length of stay [LOS], intensive therapy unit [ITU] admission, 30-day hospital readmission rate) and long-term survival.

Their results showed:

462 patients included 111 (24\%) current smokers

$55(12 \%)$ ex-smokers $<6$ weeks

$245(53 \%)$ ex-smokers $\geq 6$ weeks

$51(11 \%)$ never smokers.

The reason of selected lung cancer case was that the majority of patients usually are heavy smokers, and as the smoking has significant negative effects postoperatively it leads mainly and most commonly to pulmonary complications $[1,5,6]$. Therefore, lung cancer cases have a higher risk of postoperative pulmonary complications than any other cases.

\section{Objectives of the study}

The purpose of this study was to evaluate the duration of smoking cessation on the pulmonary state after general anesthesia and surgical procedure.

Hypothesis: Is it mandatory to stop smoking pre-operative for duration of 6 weeks?

\section{Methodology}

This study used retrospective observation; crosssectional observational study will include the following variables: adult smoker patient's age $\geq 18$ years old who are coming for elective surgery under general anaesthesia in King Fahad Specialist Hospital in Dammam Saudi Arabia in comparison with adult smoker patient's age $\geq 18$ years old who are coming for mandatory urgent surgery (bronchogenic carcinoma). We completed the patients' variables and complications in the data sheet. Data sheets coded and we had a code sheet with a code and medical record number to secure patient's confidentiality. We did not mention any personal information (name, ID number). We replaced all names by code (patient1, patient2 ...etc). Respiratory complications identified as Pneumonia, bronchospasm, emphysema, pulmonary embolism, atelectasis, bronchitis and cough. Lung cancer causes the growth of tumors that reduce a person's ability to breathe. We completed the patients' variables and complications in the data sheet. Data sheets coded and we had a code sheet with a code and medical record number to secure patient's confidentiality. We did not mention any personal information (name, ID number). We replaced all names by code (patient1, patient2 ...). Pressure controlled ventilation with PEEP was used to avoid lung injury. Fluids were managed using CVP to avoid volume overload and pulmonary edema. Postoperative pain was controlled using thoracic epidural analgesia.

\section{Sample size}

Adult smoker patients age $\geq 18$ who are coming for elective surgery under general anaesthesia in King Fahad Specialist Hospital in Dammam Saudi Arabia in comparison with adult smoker patient's age $\geq 18$ who are coming for mandatory urgent surgery (bronchogenic carcinoma).

Inclusion criteria:

- Smoker patients.

- Age $\geq 18$ years old.

- Undergoing general anesthesia.

- Elective or mandatory surgery.

- Comparison between smoker patients who stopped smoking for 6 weeks and other smoker patients who stopped smoking for less than 6 weeks at King Fahad Specialist Hospital in Dammam Saudi Arabia

\section{Exclusion criteria:}

- Age $<18$ years old.

- Non-smoker patients.

- Procedures under regional anesthesia.

Procedures with only mask ventilation (did not require airway instrumentation).

Instrument and tools of data collection: We collected the data from patient's files to find how far duration of smoking cessation affects the post-operative pulmonary complications by the patient demographic sheet.

Procedure: In this procedure, we completed the patients' variables and complications in the data sheet. Data sheets coded and we had a code sheet with a code and medical record number to secure patient's confidentiality. We did not mention any personal information (name, ID number). We replaced all names by code (patient 1 , patient 2).

Data analysis: From the data collection sheet, we applied 


\section{Anaesthesia \& Critical Care Medicine Journal}

Chi-square test in testing the association between the variables. The findings from the data collection sheet divided in the complications table. The table contains the smoker patients who got post-operative pulmonary complications and other who did not (Complications Table). We answered the concerns or question of our research according to the data collection sheet findings.

Ethical consideration: The study approved by the ethics committee of Prince Sultan Military College of Health Sciences (PSMCHS), the ministry of health, hospitals, and department of anesthesia. In addition, all participants were assured that all personal information concerning their health status would be kept confidential. We completed the patients' variables and complications in the data sheet. Data sheets coded and we had a code sheet with a code and medical record number to secure patient's confidentiality. We did not mention any personal information (name, ID number). We replaced all names by code (patient1, patient2 ...).

\section{Results}

A total of 30 smoker patients met our study inclusion criteria. Out of all, 15 smoker patients stopped smoking for 6 weeks or more. While the other 15 smoker patients stopped smoking for less than 6 weeks. A chi-square test performed to examine the association between the smoker patients who stopped smoking for 6 weeks and other smoker patients who stopped smoking for less than 6 weeks on getting postoperative pulmonary complications. The Chi square was 3.33 . The P-value was 0.07 . The relation between these variables showed an insignificant difference at $\mathrm{P}$-value $<0.05$. A 55.6\% of the smoker patients $(100 \%$ of the smoker patients stopped smoking for 6 weeks) had no post-operative pulmonary complications, and $44.4 \%$ of the smoker patients $(20 \%$ of smoker patients stopped smoking for less than 6 weeks) had post-operative pulmonary complications (Figure 1).

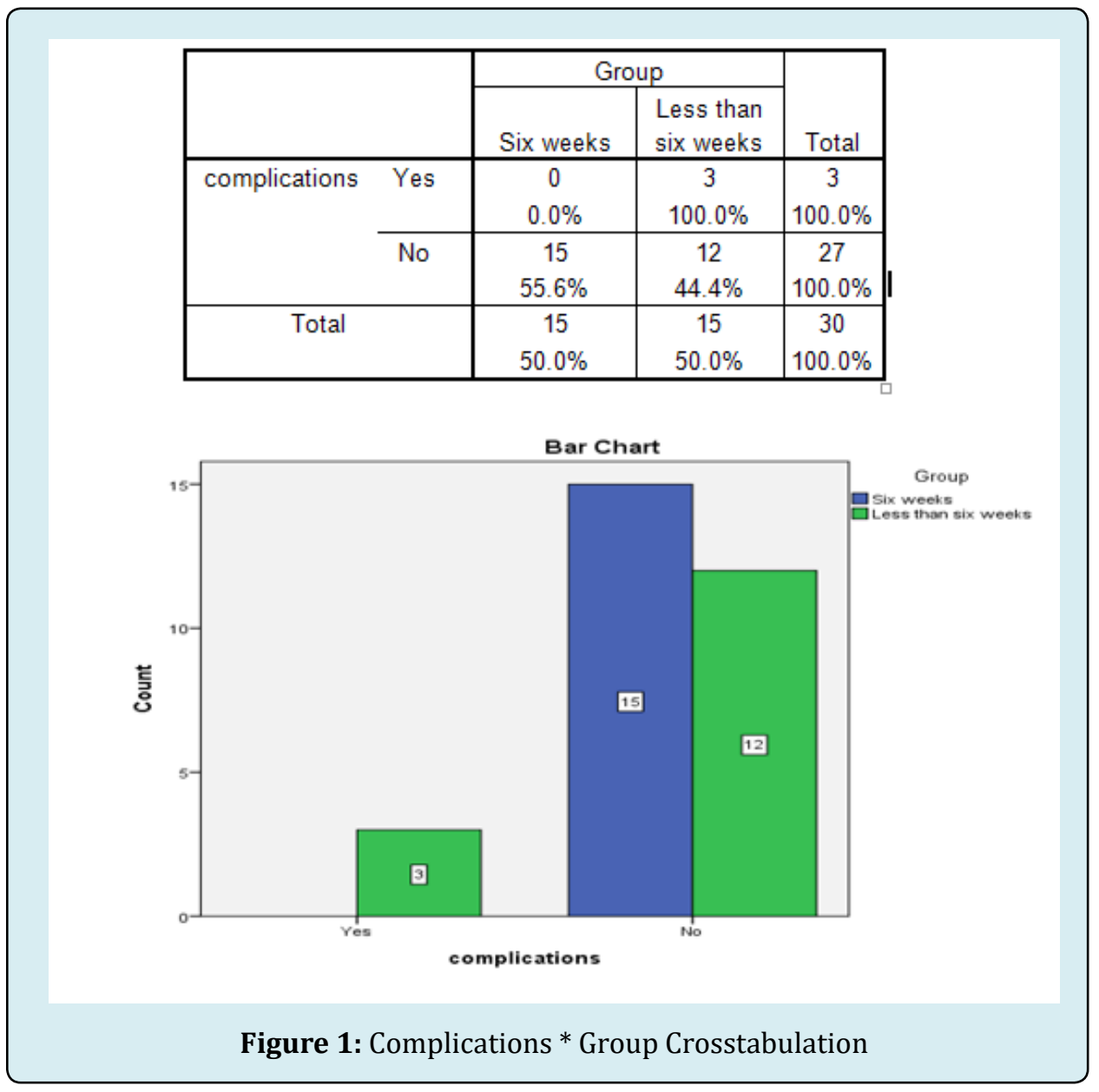

Another chi-square test performed to find out the type of post-operative pulmonary complications occurred. The Chi square was 3.33. The P-value was 0.33. The type of complications was pneumonia, cough, bronchitis and bronchospasm (Figure 2). 
Anaesthesia \& Critical Care Medicine Journal

\begin{tabular}{|rr|r|r|r|}
\hline & \multicolumn{2}{|r|}{ Group } & \\
\cline { 3 - 5 } & & Sil & $\begin{array}{r}\text { Less than } \\
\text { six weeks }\end{array}$ & Total \\
\hline types of & & 15 & 12 & 27 \\
complications & $55.6 \%$ & $44.4 \%$ & $100.0 \%$ \\
\cline { 3 - 5 } & & 0 & 1 & 1 \\
& pneumonia and & $0.0 \%$ & $100.0 \%$ & $100.0 \%$ \\
\cline { 2 - 5 } cough & 0 & 1 & 1 \\
& pneumonia and & $0.0 \%$ & $100.0 \%$ & $100.0 \%$ \\
\hline bronchitis & 0 & 1 & 1 \\
& bronchospasm & $0.0 \%$ & $100.0 \%$ & $100.0 \%$ \\
\hline & 15 & 15 & 30 \\
& Total & $50.0 \%$ & $50.0 \%$ & $100.0 \%$ \\
\hline
\end{tabular}

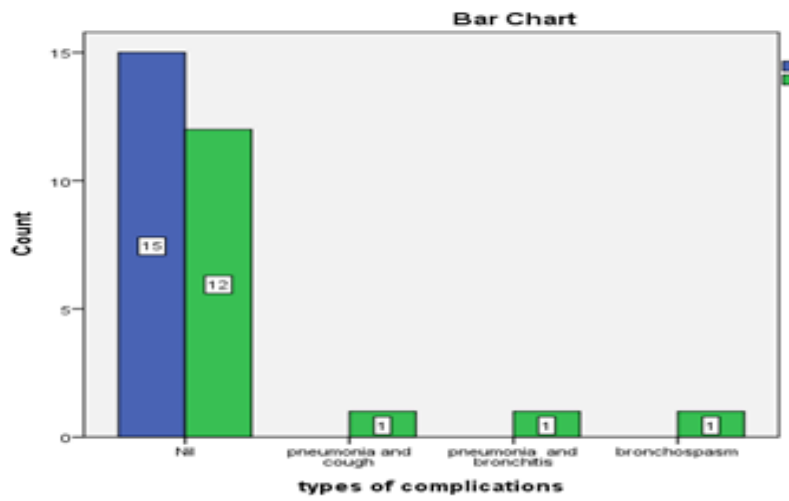

Figure 2: Types of complications * Group Crosstabulation

\begin{tabular}{|c|c|c|c|c|}
\hline & & \multicolumn{2}{|c|}{ Group } & \multirow[b]{2}{*}{ Total } \\
\hline & & $\begin{array}{c}\text { Six } \\
\text { weeks }\end{array}$ & $\begin{array}{c}\text { Less than six } \\
\text { weeks }\end{array}$ & \\
\hline \multirow[t]{4}{*}{$\begin{array}{c}\text { onset of } \\
\text { complications }\end{array}$} & & $\begin{array}{c}15 \\
55.6 \% \\
\end{array}$ & $\begin{array}{c}12 \\
44.4 \%\end{array}$ & $\begin{array}{c}27 \\
100.0 \%\end{array}$ \\
\hline & $\begin{array}{c}\text { after } 48 \\
\text { hr }\end{array}$ & $\begin{array}{c}0 \\
0.0 \%\end{array}$ & $\begin{array}{c}1 \\
100.0 \%\end{array}$ & $\begin{array}{c}1 \\
100.0 \%\end{array}$ \\
\hline & $\begin{array}{c}\text { after } 50 \\
\text { hr }\end{array}$ & $\begin{array}{c}0 \\
0.0 \%\end{array}$ & $\begin{array}{c}1 \\
100.0 \%\end{array}$ & $\begin{array}{c}1 \\
100.0 \%\end{array}$ \\
\hline & $\begin{array}{c}\text { after } 72 \\
\text { hr }\end{array}$ & $\begin{array}{c}0 \\
0.0 \%\end{array}$ & $\begin{array}{c}1 \\
100.0 \%\end{array}$ & $\begin{array}{c}1 \\
100.0 \%\end{array}$ \\
\hline \multicolumn{2}{|l|}{ Total } & $\begin{array}{c}15 \\
50.0 \% \\
\end{array}$ & $\begin{array}{c}15 \\
50.0 \%\end{array}$ & $\begin{array}{c}30 \\
100.0 \%\end{array}$ \\
\hline
\end{tabular}

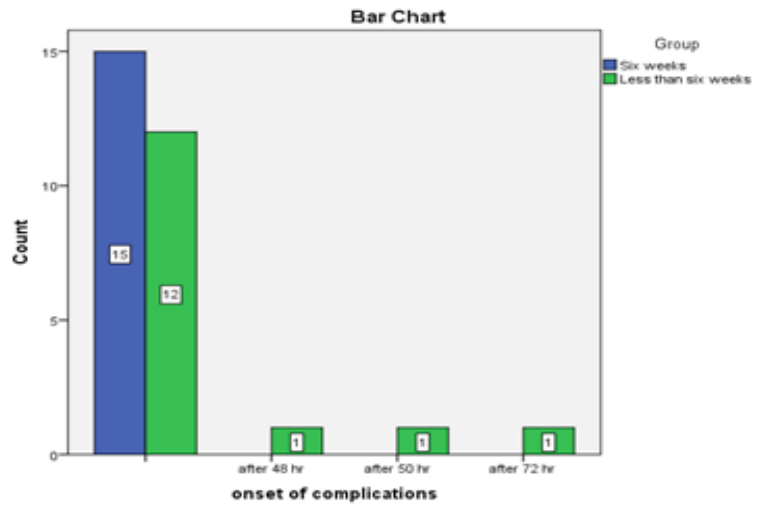

Figure 3: Onset of complications * Group Cross tabulation 


\section{Anaesthesia \& Critical Care Medicine Journal}

From Figure 3 the onset of post-operative pulmonary complications occurred after 48,50 and 72 hours in the postoperative period. Therefore, we concluded that there is no association between the mentioned variables above. A Chisquare test applied to investigate the association between duration of smoking cessation and the post-operative pulmonary complications.

\section{Discussion}

Previous studies strongly recommended smoking cessation more than six weeks. Our study aimed to determine the value of duration of preoperative smoking cessation on reducing postoperative pulmonary complications (PPCs). Our findings demonstrate that smoking cessation duration of more than six weeks before surgery has no significant value to avoid PPCs. There was an insignificant difference between smoker patients who stopped smoking for more than 6 weeks and smoker patients who stopped smoking for less than 6 weeks with a p-value of $<0.05$.

There have been a number of studies which assessed the value of duration of smoking cessation in terms of postoperative pulmonary complications $[7,8]$. The most recent study was a prospective analysis by Kozub et al. (2019), which was undertaken from 2010 till 2015 to assess the impact of timing of smoking cessation on the incidence of postoperative pulmonary complications in patients undergoing surgery for non- small cell lung cancer. Similar to our findings, duration of smoking cessation had no significant influence on frequency and/or type of PPCs. However, it should be noted that the study included twelve never smoker patients out of 286 patients, which might have introduced selection bias their study findings. Seok et al. (2013) preceded this with similar findings. Their analysis revealed that smoking was not a significant risk factor for the development of PPCs. However, both Seok et al. and Kozub et al. were limited by their study design as being only observational.

Similarly, Groth et al. (2009) found that smoking cessation before surgery did not have a significant impact on PPCs and therefore should not be a reason to delay surgery. However, the study might have been limited by reliance on self-report of patients to document the amount of tobacco they have used. Furthermore, Matsuoka et al. (2019) revealed that there was no relationship between the length of the preoperative smoking cessation period and the frequency of PPCs. The study might have been empowered by the large sample size (1248 patients), which increases the validity of their findings. However, it must be noted that this was a single institution study and might not be generalizable to the entire population irrespective of the large sample size. A case-control study by Rodriguez, et al. (2016) retrospectively reviewed database of 2139 patients who underwent anatomical lung resection. The authors stress that smoking was not recognized as a risk variable in this population of patients by risk criteria for PPCs at the moment of surgery. Therefore, it does not seem justified to postpone surgery until tobacco abstention has been achieved.

There is an area of disagreement with our findings and the literature regarding the impact of preoperative smoking cessation on PPCs. Mason, et al. (2009) and Lugg, et al. (2017) conducted observational studies to assess the effect of smoking cessation on PPCs. They showed that there was a greater frequency of PPCs in patients who continue to smoke until the date of curative-intent lung cancer surgery. Smoking increased the risk of PPCs after surgery in their studies and was slowly mitigated by preoperative cessation. However, the differences between groups in the study by Mason, et al. (2009) were not statistically significant to recommend optimal timing for smoking cessation. One critique of this study is that the prevalence of PPCs was small $(6.9 \%$ in smokers) since only significant respiratory complications were recorded, indicating that minor yet more common PPCs might have been missed. Mason et al. (2009) and Lugg et al. (2017) were limited by the retrospective nature of data collection. Randomized controlled trials are needed to confirm efficacy of smoking cessation programs.

Comparing our findings with results of other studies is difficult due to differences in study designs and difference in the definition of PPCs, which might include severe and/ or less frequent complications such as pulmonary embolism in some studies [9]. There is a paucity of literature on identifying the optimal interval of smoking cessation before surgery.

Reducing postoperative respiratory complications after cessation of smoking is believed to be linked to improving ciliary function, marcophage activity, and reducing sputum production [10]. Because these changes may take weeks or months to appear it takes a significant quantity of time to become evident to the consequences of smoking cessation [11]. Some researchers believe that the impact of smoking on the development of PPCs has been reduced due to the increase in quality of pre and postoperative management and surgery procedures [12]. Accordingly, there appears to be no need to delay surgery in order to ensure a substantial length of cessation of smoking in patients with lung cancer. Long-term cessation of smoking creates unreliable health advantages [9] but researchers consider the effect of cessation on decreasing complication risk to be ambiguous [6]. Many studies addressing this issue is retrospective in nature with small sample sizes, therefore, randomized controlled trials are strongly recommended. 


\section{Anaesthesia \& Critical Care Medicine Journal}

Some studies suggested that a few weeks of smoking cessation prior to lung surgery could lead to an unexpected high rate of PPCs $[9,13]$. Such an enhanced risk could be linked to nicotine withdrawal among those patients who quit their cigarette consumption near surgery [14].

\section{Conclusion and Recommendations}

We concluded that there is no association between smoker patients who stopped smoking for 6 weeks and other smoker patients who stopped smoking for less than 6 weeks on getting post-operative pulmonary complications. Taken together, our results in light of previous literature suggest that improvement in the perioperative care such as early ambulation and respiratory therapy could result in reduced PPCs. An implication of our study findings is that it seems unnecessary to postpone surgery to allow smoking cessation .Providing the standard respiratory care for the patients in the post-operative period will improve their condition and decrease the chance of getting post-operative pulmonary complications. Video-assisted thoracoscopic surgery techniques (VATS) are being applied more commonly which have the potential to lower the overall rate of PPCs. A greater focus on preoperative patient management, anesthesia, and surgical techniques are required to improve pulmonary function. Smoking cessation education is therefore still suggested when treating patients with lung cancer, but it seems unnecessary to dely surgery to ensure a lengthy cessation of smoking period. More research on on the efficacy of optimal time of smoking cessation needs to be undertaken before the association between smoking cessation and PPCs is more clearly understood. Until an optimal timing of smoking cessation is identified, patients should be counselled to quit smoking regardless of surgery timing.

\section{References}

1. Bradley A, Marshall A, Stonehewer L, Reaper L, Parker $\mathrm{K}$, et al. (2013) Pulmonary rehabilitation programme for patients undergoing curative lung cancer surgery. European Journal of Cardio-Thoracic Surgery 44(4): e266-e271.

2. Cooley ME, Sarna L, Kotlerman J, Lukanich JM, Jaklitsch M, et al. (2009) Smoking cessation is challenging even for patients recovering from lung cancer surgery with curative intent. Lung cancer 66(2): 218-225.

3. Gajdos C, Hawn MT, Campagna EJ, Henderson WG, Singh JA, et al. (2012) Adverse effects of smoking on postoperative outcomes in cancer patients. Annals of surgical oncology 19(5): 1430-1438.

4. Groth SS, Whitson BA, Kuskowski MA, Holmstrom AM, Rubins JB, et al. (2009) Impact of preoperative smoking status on postoperative complication rates and pulmonary function test results 1-year following pulmonary resection for non-small cell lung cancer. Lung Cancer 64(3): 352-357.

5. Kozub M, Gachewicz B, Kasprzyk M, Roszak M, Gasiorowski L, et al. (2019) Impact of smoking history on postoperative complications after lung cancer surgery-a study based on 286 cases. Kardiochirurgia i torakochirurgia pol 16(1): 13-18.

6. Lee PN, Forey BA, Coombs KJ (2012) Systematic review with meta-analysis of the epidemiological evidence in the 1900 s relating smoking to lung cancer. BMC Cancer 12: 385 .

7. Lugg ST, Tikka T, Agostini PJ, Kerr A, Adams K, et al. (2017) Smoking and timing of cessation on postoperative pulmonary complications after curative-intent lung cancer surgery. J Cardiothorac Surg 12(1): 52.

8. Mason DP, Subramanian S, Nowicki ER, Grab JD, Murthy SC, et al. (2009) Impact of smoking cessation before resection of lung cancer: a Society of Thoracic Surgeons General Thoracic Surgery Database study. The Annals of thoracic surgery 88(2): 362-371.

9. Matsuoka K, Yamada T, Matsuoka T, Nagai S, Ueda M, et al. (2019) Preoperative Smoking Cessation Period Is Not Related to Postoperative Respiratory Complications in Patients Undergoing Lung Cancer Surgery. Annals of Thoracic and Cardiovascular Surgery 25(6): 304-310.

10. Mediratta N, Poullis M (2016) Smoking status and 30-day mortality in patients undergoing pulmonary resections. Asian Cardiovasc Thorac Ann 24(7): 663-669.

11. Miskovic A, Lumb AB (2017) Postoperative pulmonary complications. BJA 118(3): 317-334.

12. Quraishi SA, Orkin FK, Roizen MF (2006) The anaesthesia preoperative assessment: an opportunity for smoking cessation intervention. J Clin Anesth 18(8): 635-640.

13. Rodriguez M, Gomez-Hernandez MT, Novoa N, Jiménez MF, Aranda JL, et al. (2016) Refraining from smoking shortly before lobectomy has no influence on the risk of pulmonary complications: a case-control study on a matched population. Eur J Cardio-Thoracic Sur 51(3): 498-503.

14. Seok Y, Hong N, Lee E (2013) Impact of smoking history on postoperative pulmonary complications: a review of recent lung cancer patients. Ann Thoracic Cardiovasc Sur 20(2): 123-128. 